\title{
Surveillance of antibiotic and analgesic use in the Oral Surgery Department of the University Dentistry Clinical Center of Kosovo
}

This article was published in the following Dove Press journal:

Therapeutics and Clinical Risk Management

I October 2015

Number of times this article has been viewed

\author{
Naim R Haliti' \\ Fehim R Haliti ${ }^{2}$ \\ Ferit K Koçani ${ }^{3}$ \\ Ali A Gashi ${ }^{4}$ \\ Shefqet I Mrasori ${ }^{3}$ \\ Valon I Hyseni ${ }^{5}$ \\ Samir I Bytyqi ${ }^{5}$ \\ Lumnije L Krasniqi ${ }^{2}$ \\ Ardiana F Murtezani ${ }^{5}$ \\ Shaip L Krasniqi ${ }^{5}$ \\ 'Department of Forensic Medicine, \\ Faculty of Medicine, University \\ of Prishtina "Hasan Prishtina", \\ 2Department of Children Dentistry, \\ University Dentistry Clinical Center \\ of Kosovo, ${ }^{3}$ Department of Oral \\ Disease, University Dentistry Clinical \\ Center of Kosovo, ${ }^{4}$ Department of \\ Oral Surgery, University Dentistry \\ Clinical Center of Kosovo, ${ }^{5}$ Institute \\ of Pharmacology and Toxicology \\ and Clinical Pharmacology, Faculty \\ of Medicine, University of Prishtina \\ "Hasan Prishtina”, Prishtina, Kosovo
}

Correspondence: Shaip L Krasniqi Institute of Pharmacology and Toxicology and Clinical Pharmacology, Faculty of Medicine, University of Prishtina "Hasan Prishtina", "Mother Teresa" Street Rrethi i Spitaleve pn, 10000 Prishtina, Kosovo

Tel +38I 385006002012

Email shaip.krasniqi@uni-pr.edu
Background: Because Kosovo has no reliable information on antimicrobial and analgesic use in dental practice, the survey reported here evaluated the antibiotic and analgesic prescriptions in the Oral Surgery Department of the University Dentistry Clinical Center of Kosovo (UDCCK).

Methods: The data of 2,442 registered patients for a 1-year period were screened and analyzed concerning antibiotic and analgesic use as per standards of rational prescription.

Results: Dentistry doctors prescribed antibiotics significantly more often than analgesics. Antibiotics were prescribed in $8.11 \%$ of all cases, while only $1.35 \%$ of total prescriptions were for analgesics. The total consumption of antibiotic drugs in the UDCCK was 4.53 Defined Daily Doses [DDD]/1,000 inhabitants/day, compared with only 0.216 DDD/1,000 inhabitants/ day for analgesics. From a total number of 117 patients, 32 patients received combinations of two antibiotics.

Conclusion: Pharmacotherapy analysis showed that the prescription rates of antibiotics and analgesics in the UDCCK are not rational in terms of the qualitative aspects of treatment. For the qualitative improvement of prescription of these drug groups, we recommend the implementation of treatment guidelines following rational standards.

Keywords: antibiotic, analgesics, Anatomic Therapeutic Classification, DDD/1,000 inhabitants/ day, rational standards

\section{Introduction}

Health professionals in all fields of health care, including dentists, are increasingly concerned with issues related to drug prescription, especially those for antimicrobial drugs. ${ }^{1-2}$ Health professionals agree that the prescription of drug therapy requires them to take an active role in the therapy, to constantly monitor patients, and qualitatively and quantitatively record outcomes to increase the efficiency and safety of these drugs. ${ }^{3-4}$ To achieve continuous monitoring of medicine prescriptions and analysis of the factors affecting the quality of therapy, more pharmacoepidemiological studies than ever before are being undertaken by researchers. These pharmacoepidemiological studies have tended to be comprehensive and have an international character, so their results will have a positive global impact on rational pharmacotherapy. ${ }^{5-6}$ The studies have presented nonrationality in the prescription of broad-spectrum antibiotics and have incited policymakers to apply restrictive measures that have improved drug-prescription standards within the national policies of various countries. ${ }^{7-8}$

Antibiotics are one of the most important groups of drugs in pharmacotherapy; the clinical significance of infections that call for use of this group of drugs is frequently described along with profiles of their side effects and their overall cost. Research into 
antibiotic usage has shown that the frequency of their irrational prescription is still unacceptably high and reflects the increased degree of microbial resistance, which has consequences for welfare and human health. ${ }^{9-11}$ In the context of clinical dental services, antibiotics and analgesics are considered the most important drugs. Therefore, it is essential to analyze the quality of these drugs to raise the standards of rational pharmacotherapy. ${ }^{12-13}$ Analyses of pharmacotherapy descriptions of these groups of drugs used in clinical dental services will be very helpful in providing feedback related to safety and cost-effectiveness of these drugs.

Results from this research will encourage the systematic process of the health system in our country to become part of regional and Pan-European initiatives for monitoring and reporting the quality of therapy. ${ }^{14}$ Therefore, the objective of the research reported here was to analyze the frequency of antibiotic and analgesic prescription in oral surgery services within the University Dentistry Clinical Center of Kosovo (UDCCK).

\section{Materials and methods}

According the standards of Ethics Committee of University Dentistry Clinical Centre of Kosova this survey is categorized in retrospective non manipulative study, using the medical data without interfering in personal data of patients. In coherence with these standards no need for approval of Ethics Committee or no need for patient's informed consent for publishing of this article. A pharmacoepidemiological retrospective study was performed using data in the medical records of registered outpatients at the Department of Oral Surgery of the UDCCK from January to December 2014.

We collected data on antibiotic and analgesic use by reviewing the prescription of antibiotics and analgesics from patient registers using the methodology of the Drug Utilization Research retrospective program. Data were collected manually on approved forms for data collection by our team, whose members have attended training on drug utilization. The World Health Organization (WHO) recommends use of the Anatomic Therapeutic Classification with Defined Daily Doses (ATC/DDD) index, ${ }^{15}$ which was designed in collaboration with the Centre for Drug Statistics Methodology for evaluating the intensity of antibiotic and analgesic use in outpatients. The ATC/DDD index provides ATC codes and DDD values for each individual drug registered. In total, 2,442 patients were registered in the department.

The data from the patient cards collected and entered in the approved database contained information regarding the age and sex of the patients, diagnosis by International Statistical Classification of Diseases and Related Health
Problems: 10th Revision (ICD-10) code, the daily dose of antimicrobial use, antimicrobial susceptibility testing and ATC antimicrobial classification, reported adverse drug effects, the trade or generic name of the drug, and numbers of drugs prescribed to each patient. ${ }^{16}$

In this study, we analyzed data using Microsoft ${ }^{\circledR}$ Excel software. The results are presented using descriptive statistics as means and percentages. Quantitative analysis was undertaken using methodology based on DDD/1,000 inhabitants/ day and ATC classification, according to WHO. ${ }^{15}$

\section{Results}

Analysis of collected data revealed that 198 patients $(8.4 \%)$ were prescribed antibiotics from the total number of 2,442 registered patients. The total antibiotic use (ATC group J01) at the UDCCK was 4.53 DDD/1,000 inhabitants/day, while the total analgesic use was only $0.216 \mathrm{DDD} / 1,000$ inhabitants/day. There were 255 individual prescriptions of antibiotics registered to these treated patients. From the total number of patients who took antibiotics, $51.52 \%$ were female. In total, 142 patients were prescribed one antibiotic while 56 patients were prescribed a combination of two antibiotics (amoxicillin + clavulanic acid in 54 patients and amoxicillin + metronidazole in two patients).

From the total number of antibiotics given $(n=255)$, $43.1 \%$ were prescribed by generic name, whereas $56.9 \%$ were prescribed by trade name. Analyzing prescription preferences, we discovered that $97.25 \%$ of the antibiotics were from a list of essential medicines approved by the Ministry of Health of Kosovo (the list of medical products and sanitary medical consumables purchased by the Kosovo Consolidated Budget to meet the needs in all levels of health care). Our results show that prescription of antibiotics in any case is empirical and not based on microbiological sensitivity tests, because there was no data for antibiograms.

Based on the collected data, we saw the number of analgesic drugs described for the total number of patients was obviously fewer than the number of antibiotics. Of the total number of registered patients $(n=2,442)$, only 33 patients $(1.35 \%)$ were prescribed an analgesic.

No statistically significant difference was found between males and females regarding the prescription of antibiotics and analgesics (Table 1).

Table 2 depicts the total number of patients prescribed antibiotics and the differences between age subgroup. Antibiotic use among the age subgroups differed significantly $\left(\chi^{2}\right.$ test $\left.=20.80, P<0.05\right)$, ranging from $3.96 \%$ in the subgroup of patients aged over 60 years to $10.85 \%$ in the subgroup aged $19-40$ years. 
Table I Structure of prescription - general data $(n=2,442)$

\begin{tabular}{llllllll}
\hline Drug group & Treated patients (n [\%]) & Prescriptions (n) & $\begin{array}{l}\text { Treated patients } \\
(\mathbf{n}[\%])\end{array}$ & Male/female & Statistical significance \\
\cline { 3 - 6 } & & Males & Females & \\
\hline Antibiotics & $198(8.11)$ & 255 & $96(8.40)$ & $102(7.85)$ & $\chi^{2}$ test $=0.2439, P=0.62$ & Not significant at $P<0.05$ \\
Analgesics & $33(1.35)$ & 33 & $13(1.14)$ & $20(1.54)$ & $\chi^{2}$ test $=0.73, P=0.39$ & Not significant at $P<0.05$ \\
\hline
\end{tabular}

For total number of patients $(\mathrm{n}=2,442)$ the dominant age group is 19 to 40 years ( $44.14 \%$ of total patients) and 41 to 60 years old (30.02\% of total patients). For all patients with antibiotics ( $\mathrm{n}=255)$, dominant age group is a group of patients $19-40$ years $(69.8 \%)$, followed by a group of patients $41-60$ years old (18.82\%).

Figure 1 shows the antimicrobials used, by subgroup, by DDD/1,000 inhabitants/day. From the general structure of antibiotic classification, four subclasses of antibiotics were prescribed. Beta-lactam antibiotics (J01C) were the most used antibiotic subgroup (3.86 DDD). Other antibacterial, macrolide, and other beta-lactam antibiotic subgroups were used in low proportions $(0.53,0.13$, and 0.01 , respectively).

Figure 2 presents the antibiotic consumption according to individual antimicrobial. Six individual antibiotics were prescribed. The most prescribed antibiotics were amoxicillin + clavulanic acid, which were significantly consumed, approximately six times more often than metronidazole, the secondmost-prescribed antibiotic. Compared with other individual antibiotics, the rate of use of amoxicillin + clavulanic acid was several dozen times more.

Analgesic drugs had a relatively low consumption value, from 0.01 to $0.13 \mathrm{DDD} / 1,000$ inhabitants/day (Figure 3). Four analgesic drugs were prescribed from the classification of all analgesic drugs; significantly, ibuprofen was prescribed several times more often than dexketoprofen, metamizole sodium, and paracetamol.

Low total analgesic use does not reflect the real prescription rate of analgesics in this department, due to the fact that sometimes dental doctors advise, but do not prescribe, patients to take an analgesic in case of pain. As none of these cases is documented by a prescription, this results in a low apparent rate of analgesic use.

No adverse events from drugs were reported for any registered patient.
In Figure 4, we present a classification of diseases according to the ICD-10 and based on study patient groups. According to this classification, the group of patients who did not receive antibiotics predominated in the categories of ICD-10 disease codes K08 (937 patients), K04 (507 patients), and K05 (415 patients), whereas the other categories had fewer numbers. For the group of patients who received antibiotics, we identified nine categories of diseases, dominated by the diseases of pulp and periapical tissues ( 83 patients), gingivitis and periodontal diseases (44 patients), and other disorders of teeth and supporting structures (29 patients) categories, whereas the number of patients in other disease categories was significantly fewer. However, the patients who received analgesics were most prevalent in the diseases of pulp and periapical tissues category, with almost half of all patients in this category including those who received analgesics.

\section{Discussion}

Data from the survey show that the prescription of antibiotics in the dental clinic practice of the UDCCK substantially differs from international prescription trends in dental clinical practice. ${ }^{17}$ A relatively low percentage of all registered outpatients of oral surgery at the UDCCK were prescribed antibiotics.

In the Oral Surgery Department, we depict the low prescription rate of antibiotics (a quantitative rational prescription) but not rational choice of appropriate individual antibiotic (inadequate qualitative prescription). Penicillin is still the recommended therapy for the treatment of odontogenic infections according to the literature and treatment protocols. $^{18}$

Our analysis of the prescription of other beta-lactam antimicrobials showed that the most prescribed antibiotic was the combination of amoxicillin + clavulanic acid rather than amoxicillin or other antibiotics from other groups.

Table 2 Structure of treated patients with antibiotics by age subgroup $(n=2,442)$

\begin{tabular}{llll}
\hline Age subgroup & Total patients $(\mathbf{n})$ & With antibiotics $(\mathbf{n}[\%])$ & Statistical significance \\
\hline$<18$ years & 177 & $15(8.47)$ & $\chi^{2}$ test $=20.80, P=0.000 I 16$ \\
$19-40$ years & 1,078 & $117(10.85)$ & Significant at $P<0.05$ \\
$41-60$ years & 733 & $48(6.55)$ & \\
$>60$ years & 454 & $18(3.96)$ & \\
\hline
\end{tabular}




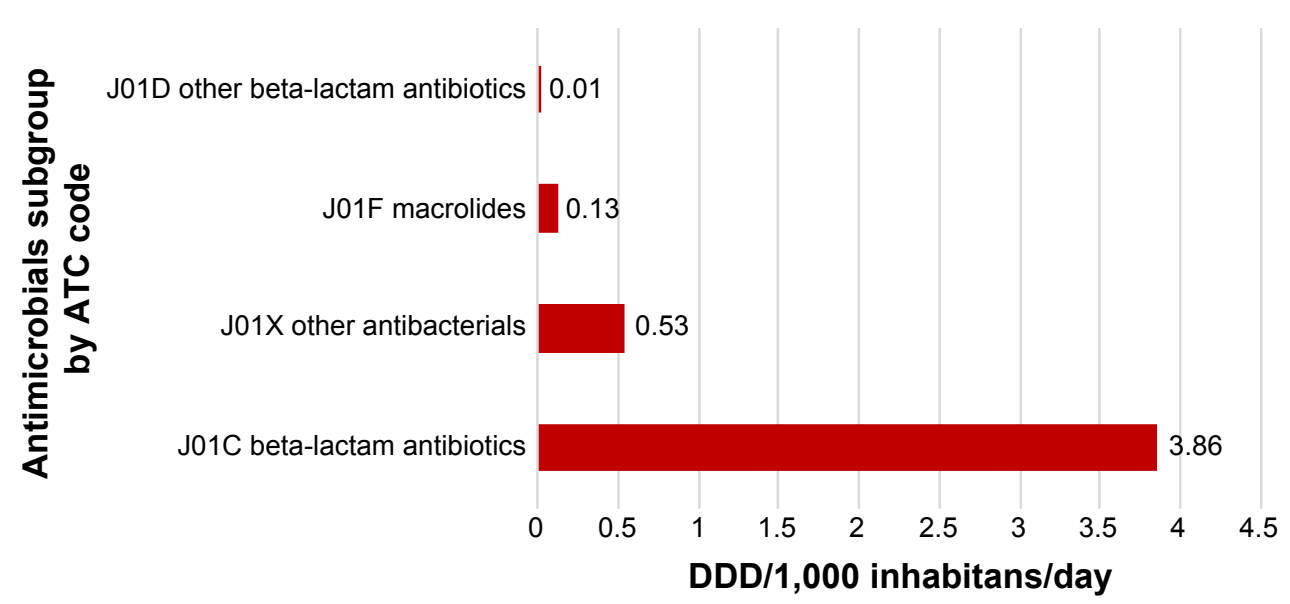

Figure I Utilization of antibacterials for systemic use - J0I (World Health Organization Anatomic Therapeutic Classification [ATC] Level 3) by ATC System with Defined Daily Doses (DDD) methodology.

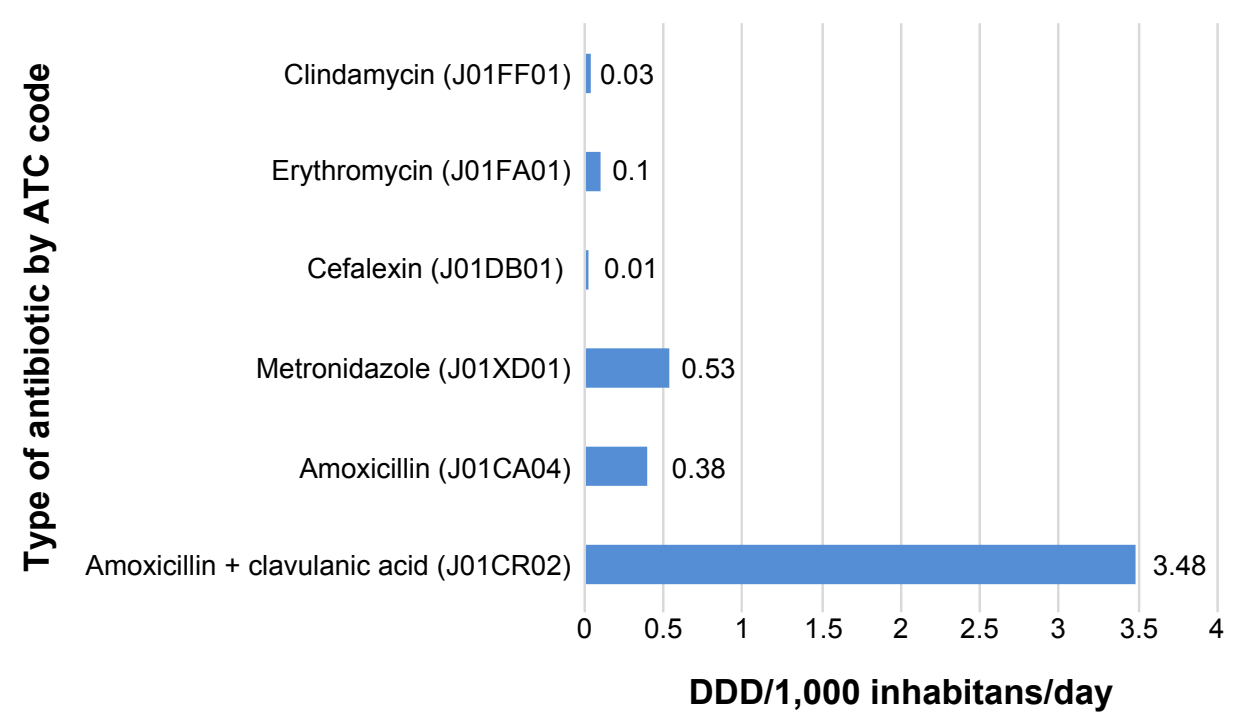

Figure 2 Utilization of individual antibacterials for systemic use (World Health Organization Anatomic Therapeutic Classification [ATC] Level 5) by ATC System with Defined Daily Doses (DDD) methodology.

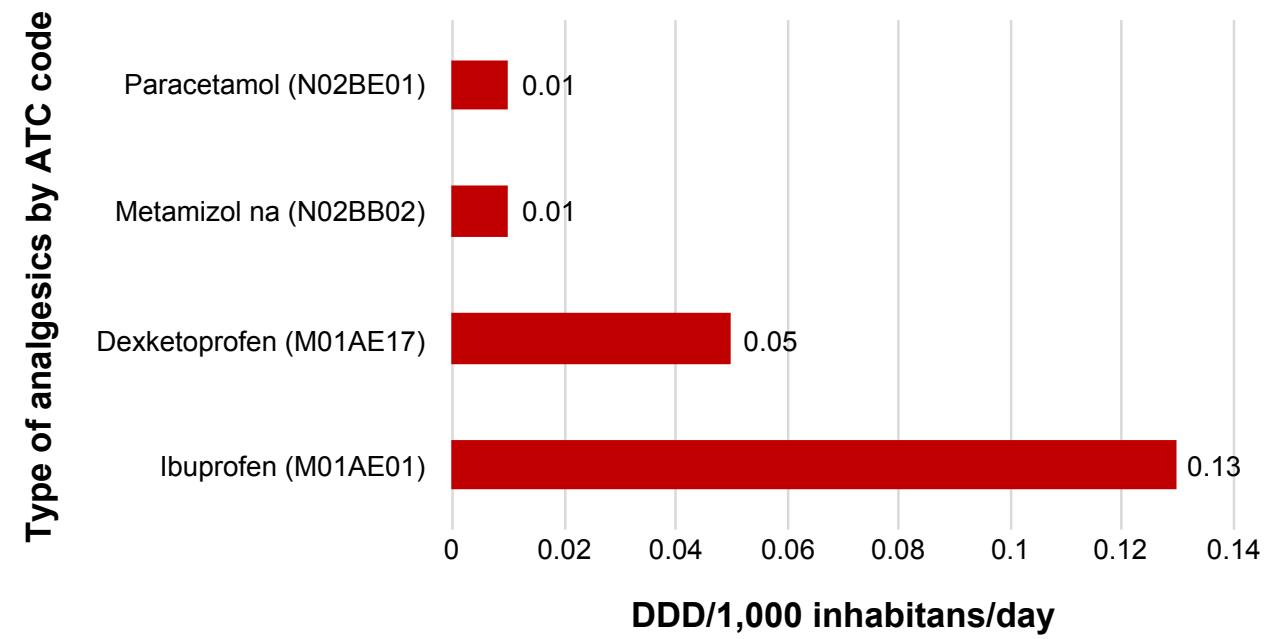

Figure 3 Utilization of individual analgesics (World Health Organization Anatomic Therapeutic Classification [ATC] Level 5) by ATC System with Defined Daily Doses (DDD) methodology. 


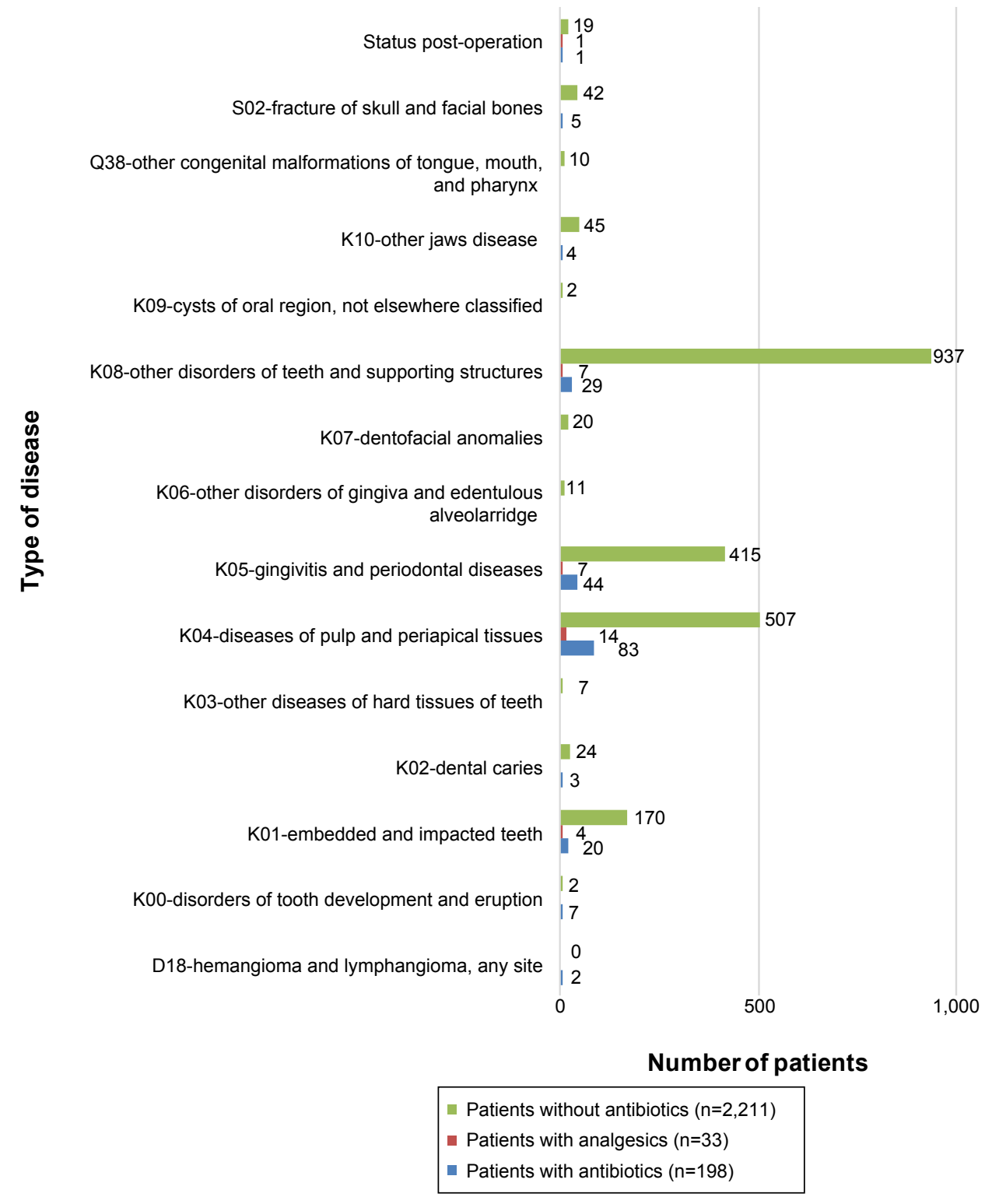

Figure 4 International Statistical Classification of Diseases and Related Health Problems: IOth Revision classification of disease by number of patients and modality of prescription of antibiotics and analgesics $(n=2,442)$.

This finding represents nonrational antibiotic prescription, considering that one effective clinical study reported that there was no difference between amoxicillin and amoxicillin + clavulanic acid in treating infections successfully. ${ }^{19}$

The prescribed antibiotics described in our survey differ from those prescribed in dental practice in Norway, where the most prescribed antibiotics were those with a narrow spectrum, such as metronidazole and phenoxymethylpenicillin. According to the authors of that study, this shows that a low level of microbial resistance still enables the clinical efficacy of narrow-spectrum antibiotics. ${ }^{20}$

During the comparison of antibiotic prescription patterns in this survey with those in dental practices of different countries, we noticed heterogeneity in the structure of the prescription profiles in other countries. We believe this to be due to the treatment protocols approved by the medical centers or health care systems and the microbial resistance profiles reported in the respective states. The analysis of prescribed antibiotics in other countries showed that dental doctors mostly prefer the broad-spectrum antibiotics from the penicillin group, such as amoxicillin. ${ }^{21-22}$ An analysis of prescribed antibiotics in the community of dentists in Spain indicates the preparation of amoxicillin + clavulanic acid as the most commonly prescribed antibiotic, comprising $38.7 \%$ of all antibiotics prescribed, and this finding matches the results regarding antibiotic use in our research. ${ }^{23}$ The total number of individual antibiotics found prescribed in our survey (six) is fewer than the number of individual 
antibiotics reported by Roda et al $(2007)^{23}$ as being prescribed in Valencia, Spain; the eleven antibiotics prescribed by dentists in Norway; ${ }^{20}$ and the 13 antibiotics reported in a paper published in Indonesia. ${ }^{24}$

The rate of analgesic drug prescription found in our survey was very low compared with that found in similar study. (1.35\% vs $86.00 \%) .{ }^{25}$ In most cases, analgesics have a higher rate of prescription than antibiotics, whereas, in our case, prescriptions of antibiotics dominated analgesics $(8.11 \%$ vs $1.35 \%){ }^{26}$

There are limitations in this study. The dental doctors in most of the cases did not prescribe analgesics, but advised patients to take analgesics in case of pain. We did not register these advices as prescriptions, and we suppose this may have contributed to the low rate of analgesic prescription. We suggest this pattern of prescription is a consequence of the absence of approved treatment protocols in the relevant department of the UDCCK, meaning that patients are enabled to take analgesics as needed and to purchase them from community pharmacies, with these uses not recorded in patient records. When analyzing individual analgesics, we found the most prescribed preparation was ibuprofen, which fits with the results of Kulkarni et al in which the authors, in their analysis of the prescription of analgesics, found diclofenac sodium as the most prescribed preparation. ${ }^{25}$ The use of dexketoprofen is not usual in dental practice and its prescription may be influenced by the marketing activities of private pharmaceutical companies in our country.

Analysis of the prescription of antibiotic by generic name is irrational according to WHO standards, which requires the description of drugs by generic name to achieve the optimum rate of $100 \%{ }^{27}$

During our analysis of the prescriptions, we noticed dentists were highly likely to prescribe antibiotics from the essential drug list approved by the Ministry of Health. During analysis of the indicators for prescribing antibiotics and analgesics, we noticed appropriate use in quantitative but not in qualitative aspects of their prescription. The prescription of antibiotics in all cases is empiric and usually a broad-spectrum therapy. Under these conditions, the literature states that prescribing an antibiotic without susceptibility testing can promote bacterial resistance. ${ }^{28}$ The lack of approved official guidelines for antibiotic and analgesic use means that dental doctors may select antibiotics based on personal preference. Moreover, as there are no regulations in our country governing the marketing activities of pharmaceutical companies, the marketing activities of these companies may impact the prescribing habits of dental doctors for antibiotics and other drugs.
The missing reports of any drug adverse effects show that the pharmacovigilance system is not well established and this requires improvement.

\section{Recommendations}

To improve the quality of prescription therapy in Kosovo dentistry practice, we recommend an approved protocol for antibiotic treatment, according to data reported on microbial resistance. In addition, we recommend dental doctors take a more active role in advising and prescribing analgesics in order to monitor and to record this therapy.

\section{Disclosure}

The authors report no conflicts of interest in this work.

\section{References}

1. Williams D. Monitoring medicines use: the role of the clinical pharmacologist. Br J Clin Pharmacol. 2012;74:685-690.

2. Luo X, Cappelleri JC, Frush K. A systematic review on the application of pharmacoepidemiology in assessing prescription drug-related adverse events in pediatrics. Curr Med Res Opin. 2007;23:1015-1024.

3. Shekar V, Singh M, Shekar K, Brennan P. Clinical negligence and duty of candour. Br J Oral Maxillofac Surg. 2011;49:593-596.

4. Chamberlain CJ, Koniaris LG, Wu AW, Pawlik TM. Disclosure of "nonharmful" medical errors and other events: duty to disclose. Arch Surg. 2012;147:282-286.

5. Zarb P, Goossens H. European Surveillance of Antimicrobial Consumption (ESAC): value of a point-prevalence survey of antimicrobial use across Europe. Drugs. 2011;71:745-755.

6. Lewis M. Why we must reduce dental prescription of antibiotics: European Union Antibiotic Awareness Day. Br Dent J. 2008;10:205.

7. Thuonga MS F. Appropriate use of restricted antimicrobial agents in hospitals: the importance of empirical therapy and assisted re-evaluation. J Antimicrob Chemother. 2000;46:501-508.

8. Oberoi SS, Dhingra C, Sharma G, Sardana D. Antibiotics in dental practice: how justified are we. Int Dent J. 2015;65:4-10.

9. WHO. Antimicrobial resistance: global report on surveillance 2014. Geneva: WHO; 2014.

10. Kern WV, de With K. Rationale Antibiotikaverordnung. Mehr Herausforderungen als Erfolge. [Rational antibiotic prescribing. Challenges and successes]. Bundesgesundheitsblatt Gesundheitsforschung Gesundheitsschutz. 2012;55:1418-1426.

11. Dar-Odeh NS, Abu-Hammad OA, Al-Omiri MK, Khraisat AS, Shehabi AA. Antibiotic prescribing practices by dentists: a review. Ther Clin Risk Manag. 2010;6:301-306.

12. Krishnan V. Orthodontic pain: from causes to management - a review. Eur J Orthod. 2007;29:170-179.

13. Cherry WR, Lee JY, Shugars DA, White RP Jr, Vann WF Jr. Antibiotic use for treating dental infections in children: a survey of dentists' prescribing practices. J Am Dent Assoc. 2012;143:31-38.

14. Versporten A, Bolokhovets G, Ghazaryan L, et al. Antibiotic use in eastern Europe: a cross-national database study in coordination with the WHO Regional Office for Europe. Lancet Infect Dis. 2014;14:381-387.

15. WHO. WHO Collaborating Centre for Drug Statistics Methodology. Guidelines for ATC classification and DDD assignment 2013. Geneva: WHO; 2012.

16. WHO. International Statistical Classification of Diseases and Related Health Problems 10th Revision. Geneva: WHO; 2014.

17. Ramu C, Padmanabhan T. Indications of antibiotic prophylaxis in dental practice-Review. Asian Pac J Trop Biomed. 2012;2:749-754.

18. Swift JQ, Gulden WS. Antibiotic therapy - managing odontogenic infections. Dent Clin North Am. 2002;46:623-633, vii. 
19. Kuriyama T, Absi EG, Williams DW, Lewis MA. An outcome audit of the treatment of acute dentoalveolar infection: impact of penicillin resistance. Br Dent J. 2005;198:759-763; discussion 4; quiz 78.

20. Al-Haroni M, Skaug N. Incidence of antibiotic prescribing in dental practice in Norway and its contribution to national consumption. J Antimicrob Chemother. 2007;59:1161-1166.

21. Palmer NO, Martin MV, Pealing R, Ireland RS. An analysis of antibiotic prescriptions from general dental practitioners in England. J Antimicrob Chemother. 2000;46:1033-1035.

22. Jaunay T, Sambrook P, Goss A. Antibiotic prescribing practices by South Australian general dental practitioners. Aust Dent J. 2000;45: 179-186; quiz 214.

23. Roda R, Bagan JV, Bielsa JM, EC P. Antibiotic use in dental practice. a review. Med Oral Patol Oral Cir Bucal. 2007;12:186-192.

24. Rachmawati. Antibiotic utilization in a Dental Teaching Hospital in Yogyakarta, Indonesia. Science Journal of Clinical Medicine. 2014;3: $37-42$.
25. Kulkarni MD, Baig MS, Hussaini SA, Doifode SM. Drug utilization pattern in OPD of government dental college and hospital, Aurangabad. International Journal of Basic and Clinical Pharmacology. 2010;2:69-70.

26. Patel SS, Mark A, Desai H. Drug utilization pattern of analgesics in various departments of a tertiary care teaching rural dental hospital. Int J Basic Clin Pharmacol. 2014;3:804-806.

27. WHO. How to investigate drug use in health facilities: selected drug use indicators. Geneva, World Health Organization, EDM Research Series No. 007. 1993.

28. Goossens H. Antibiotic consumption and link to resistance. Clin Microbiol Infect. 2009;15 Suppl 3:12-15.

\section{Publish your work in this journal}

Therapeutics and Clinical Risk Management is an international, peerreviewed journal of clinical therapeutics and risk management, focusing on concise rapid reporting of clinical studies in all therapeutic areas outcomes, safety, and programs for the effective, safe, and sustained use of medicines. This journal is indexed on PubMed Central, CAS,
EMBase, Scopus and the Elsevier Bibliographic databases. The manuscript management system is completely online and includes a very quick and fair peer-review system, which is all easy to use. Visit http://www.dovepress.com/testimonials.php to read real quotes from published authors.

Submit your manuscript here: http://www.dovepress.com/therapeutics-and-clinical-risk-management-journal 